\title{
28 Research Square \\ TaxaTarget: Fast, Sensitive, and Precise Classification of Microeukaryotes in Metagenomic Data
}

Seth Commichaux ( $\sim$ Seth.Commichaux@fda.hhs.gov )

United States Food and Drug Administration

Kiran Javkar

University of Maryland, College Park

Harihara Subrahmaniam Muralidharan

University of Maryland, College Park

Padmini Ramachandran

United States Food and Drug Administration

Andrea Ottesen

United States Food and Drug Administration

Hugh Rand

United States Food and Drug Administration

Mihai Pop

University of Maryland, College Park

\section{Research Article}

Keywords: Eukaryotes, eukaryome, metagenomics, supervised learning, taxonomic classification

Posted Date: December 28th, 2021

DOI: https://doi.org/10.21203/rs.3.rs-1186624/v1

License: (c) (1) This work is licensed under a Creative Commons Attribution 4.0 International License.

Read Full License 


\section{Abstract}

\section{Background}

Microbial eukaryotes are nearly ubiquitous in microbiomes on Earth and contribute to many integral ecological functions. Metagenomics is a proven tool for studying the microbial diversity, functions, and ecology of microbiomes, but has been underutilized for microeukaryotes due to the computational challenges they present. For taxonomic classification, the use of a eukaryotic marker gene database can improve the computational efficiency, precision and sensitivity. However, state-of-the-art tools which use marker gene databases implement universal thresholds for classification rather than dynamically learning the thresholds from the database structure, impacting the accuracy of the classification process.

Results

Here we introduce taxaTarget, a method for the taxonomic classification of microeukaryotes in metagenomic data. Using a database of eukaryotic marker genes and a supervised learning approach for training, we learned the discriminatory power and classification thresholds for each 20 amino acid region of each marker gene in our database. This approach provided improved sensitivity and precision compared to other state-of-the-art approaches, with rapid runtimes and low memory usage. Additionally, taxaTarget was better able to detect the presence of multiple closely related species as well as species with no representative sequences in the database. One of the greatest challenges faced during the development of taxaTarget was the general sparsity of available sequences for microeukaryotes. Several algorithms were implemented, including threshold padding, which effectively handled the missing training data and reduced classification errors. Using taxaTarget on metagenomes from human fecal microbiomes, a broader range of genera were detected, including multiple parasites that the other tested tools missed.

\section{Conclusion}

Data-driven methods for learning classification thresholds from the structure of an input database can provide granular information about the discriminatory power of the sequences and improve the sensitivity and precision of classification. These methods will help facilitate a more comprehensive analysis of metagenomic data and expand our knowledge about the diverse eukaryotes in microbial communities.

\section{Background}

Eukaryotes, bacteria, and archaea comprise the domains of life on Earth (according to our current understanding). Well known eukaryotes, like plants and animals, belong to two small branches on a much larger phylogenetic tree of mostly uncharacterized microscopic eukaryotes, i.e., microeukaryotes. Some microeukaryotes are well known because they benefit humans. For example, Saccharomyces cerevisiae (baker's yeast) and Aspergillus sojae are involved in the creation of fermented foods, drinks, and spices such as beer, bread, miso, and soy sauce. Others, like Plasmodium falciparum, Trypanosoma 
brucei, and Cyclospora cayetanensis are infamous for causing human diseases like malaria, sleeping sickness and food poisoning outbreaks, respectively. However, studies estimate that less than $5 \%$ of the predicted millions of species of microeukaryotes are currently characterized [1-3]. Furthermore, the eukaryotic tree of life is still expanding as whole new supergroups are being discovered [4].

Microeukaryotes do not exist in isolation, rather they are ecologically integral members of diverse microbial communities (alongside bacteria, archaea, viruses) i.e., microbiomes, found nearly everywhere on Earth. For example, phytoplankton, a mixture of photosynthetic microorganisms floating in oceans and freshwater bodies, are responsible for nearly half of all carbon fixation and primary production on Earth $-70 \%$ is performed by microeukaryotic groups i.e., Diatoms, Coccolithophores, and Chlorophytes [5]. In the human gut microbiome, some species of Blastocystis and Entamoeba, once strictly considered parasites, might promote healthy and diverse assemblages of bacterial species. Further, recent studies have shown that many parasitic microeukaryotes might be commensals, with no effect on health, or even mutualists [6-11], depending upon the microbial background. There is even evidence that the absence of some parasitic microeukaryotes might be a contributing factor to the development of autoimmune diseases $[9,10]$.

Metagenomics-the sequencing of DNA extracted directly from microbial communities-provides a means for studying the microbial diversity, functional potential, and ecology of microbiomes.

Traditionally, metagenomic analyses involve aligning the short DNA sequences output by sequencing (i.e., reads) to a reference database of sequences (e.g., genomes or genes) with known taxonomy and/or functions, and transferring the annotations if sequence similarity is high. Although leading to an explosion of knowledge about microbial communities [12], most metagenomic studies have focused on bacteria, with relatively few studies (although a growing number) focusing on microeukaryotic groups such as fungi and protists $[9,13-15]$.

The metagenomic study of microeukaryotes has lagged for a variety of reasons. First, there is a general sparsity of sequenced genomes and transcriptomes, with more eukaryotic species represented in protein databases, e.g., 40,000 species in the UniRef100 database, than genome databases, e.g., 4,000 species in the National Center for Biotechnology information (NCBI) Assembly database. Combined, this is still orders of magnitude fewer species than the millions estimated to exist [1, 2]. Sparse reference sequences can negatively impact the performance of tools that use sequence similarity for classification. For example, metagenomic reads from a novel species might not align to any sequences in the reference database of a tool and thus go undetected.

Microeukaryotes often occur at low abundance in microbial communities with genomes that are orders of magnitude larger than bacteria $[16,17]$. Ideally, all the genomes in a metagenome could be directly reconstructed (i.e., assembled) from the reads. However, the algorithmic challenges of assembly, the limitations of current sequencing technologies, and the general sparsity of eukaryotic reads per metagenome, often results in few or no overlapping reads to construct longer eukaryotic sequences (i.e., contigs). Microeukaryotic contigs, longer than $3,000 \mathrm{bp}$, can sometimes be assembled, given sufficient 
depth of coverage [18], and classified as eukaryotic based upon k-mer composition with high accuracy [19]. However, current bioinformatic approaches for characterizing microeukaryotes tend to rely upon metagenomic reads for analysis.

For large eukaryotic genomes (sometimes billions of base pairs), mapping metagenomic reads directly can be computationally expensive in terms of runtime and memory. Additionally, the assemblies of eukaryotic genomes are frequently contaminated with bacteria, potentially leading to classification errors [20-22]. A more robust and scalable solution is the use of marker sequence databases such as the clade-specific genes used in MetaPhlan3 [23], universally present sequences such as the cytochrome oxidase (COI) gene or ribosomal subunits (as used by Metaxa2 [24]), or single copy orthologs (as used by EukDetect $[22,25])$. The latter have shown high specificity for eukaryotes with a strong phylogenetic signal $[18,25]$. A drawback for using a database of marker sequences is that the combined length of the marker sequences is usually a small fraction of any eukaryotic genome, potentially resulting in low sensitivity when employed in metagenomic data.

A general challenge for tools using sequence similarity for taxonomic classification is that sequences, and regions of sequences, can have variable levels of discriminatory power because they evolve at different rates [26]. For example, regions of the $18 \mathrm{~S}$ ribosomal subunit (rRNA), a eukaryotic marker sequence used by Metaxa2 for taxonomic classification, are known to lack discriminatory power at the species, genus, and sometimes even the family or broader levels for some eukaryotic groups [3]. One consequence is that a metagenomic read can align equally well to a sequence in multiple species, providing insufficient evidence for classification at the species level. EukDetect addressed this problem for eukaryotic marker genes sharing conserved regions with bacteria (a common source of classification errors) by removing marker genes from its database if bacterial reads mapped, or if a Hidden Markov Model (HMM) used for eukaryotic gene annotation predicted genes in bacterial genomes [22] (note, this strategy was not extended to other groups like archaea and viruses). This reduced the number of marker gene families in the EukDetect database from 255 to 214. A drawback of discarding entire eukaryotic marker gene families, due to conserved regions in bacteria, is that all the non-conserved regions, which are potentially informative for eukaryotic taxonomic classification, will also get discarded.

It is common for taxonomic classification approaches to implement universal alignment score cutoffs as a simple method for classifying metagenomic reads mapped to database sequences. However, this also ignores that sequences can evolve at different rates and can have variable discriminatory power. EukDetect implements such an approach to classify species, as does Metaxa2 which assigns universal cutoffs for the classification of each taxonomic rank from Kingdom to species [24]. CCMetagen also assigns universal cutoffs (from phylum to species), but it implements a slightly more sophisticated approach, i.e., using classification thresholds learned from the clustering of fungal internal transcribed spacer (ITS) and ribosomal subunit marker sequences to assess the taxonomic relationship between metagenomic reads and sequences in the NCBI Nucleotide database [27]. Although the thresholds are learned for specific markers with a data-driven approach, they end up being applied to all sequences in its database which might evolve at different rates. 
As an alternative, supervised learning approaches have been developed for dynamically determining thresholds using marker genes [28] and for the discovery of protein families [29] in metagenomic data; however, these approaches were developed for bacteria using bacterial marker genes. Supervised learning methods use labeled training data to learn functions for classifying new examples. For example, MetaPhyler [28] predicts the taxonomic relatedness (from phylum to species) of metagenomic reads aligned to marker genes. It learns the cutoffs for classification thresholds by training on the scores of reads, from the genomes of known taxa, aligned to the marker genes in its database. This approach automatically assesses if sequences contain enough information for classification at different taxonomic levels. However, a limitation of MetaPhyler is that it builds classifiers for whole genes, treating all regions of a gene as having equal classification power. ROCker [29] introduced a method for assessing if metagenomic reads were sequenced from specific gene families using sliding window, region-specific classifiers. Although a functional profiling tool, we thought the approach could be successfully adapted for taxonomic classification.

Here, we present a metagenomic taxonomic profiling tool, taxaTarget, specifically designed for microeukaryotes. We show that taxaTarget is computationally efficient, with higher sensitivity, and often higher specificity than similar state-of-the-art tools using marker sequence databases (i.e., Metaxa2 and EukDetect, MetaPhlan3 was not included because it only had 122 eukaryotic species as of release mpa_v30)-even for species not represented in the database. Using a similar database of single copy orthologs as EukDetect, we show how a supervised learning approach automates the assessment of which genes and regions are most informative for classification, reducing the need for manual curation, and retaining more sequences in the database. Further, the supervised learning approach of taxaTarget replaces universal classification thresholds (i.e., universally applied to all sequences in a database) with classifiers tuned by region in a data-driven manner. One challenge for implementing a supervised learning approach was the scarcity of training data for microeukaryotes which initially led to many classification errors; however, we were able to implement several novel solutions which effectively handled errors caused by missing data.

\section{Methods/ Materials}

\section{Definitions}

Precision $=$ True Positives $/$ (True Positives + False Positives $)$

Sensitivity $=$ True Positives $/$ (True Positives + False Negatives)

False Positive Rate $=$ False Positives $/$ (False Negatives + True Negatives)

Accuracy $=($ True Positives + True Negatives $) /($ True Positives + True Negatives + False Positives + False Negatives) 


\section{Workflow and implementation}

The workflow of taxaTarget is shown in Figure 1. It is written in Python3 (Python $\geq 3.6$ ) and executed via command line. It utilizes a custom database of eukaryotic marker genes, the trained classifiers, and the NCBI taxonomy. It accepts as input raw metagenomic sequencing reads in fastq format. The reads are first mapped to the database with Kaiju (v1.8.0) [30] to quickly identify reads that might be sequenced from eukaryotic marker genes. The binned reads are then more sensitively aligned with Diamond (v2.0.11) [31] to the marker genes. The Diamond output is then provided as input to taxon-specific classifiers trained on a curated database of eukaryotic sequences. Finally, the results are aggregated and the taxonomic profile of the metagenomic sample is generated.

The taxonomic classification results are output in four tab-delimited files: 1) classified_reads.txt provides the initial classification results for individual reads that get classified as eukaryotic-this allows users to explore the raw results before filtering; 2) marker_gene_read_counts_per_taxa.txt is a matrix of read counts per marker gene for all eukaryotic lineages detected in the sample; 3) final_read_classifications.txt provides the final classification results for all reads included in the taxonomic profile; 4)

Taxonomic_report.txt provides the final aggregate taxonomic profile (i.e. read counts per taxonomic lineage and the number of marker genes with mapped reads) of the sample provided.

\section{Building the marker gene database}

The database of taxaTarget was built from protein sequences extracted from the UniRef100 database of UniProt (release-2021_02) [32] to maximize the breadth of species detectable. The UniRef100 database was used, instead of UniProt, because it removes identical sequences and assigns the lowest (most recent) common ancestor to the representative sequences in the database. The $\sim 2.0 \times 10^{8}$ protein sequences in the UniRef100 database were downloaded on April 15, 2021. Approximately $6.4 \times 10^{7}$ eukaryotic proteins were extracted and assigned a taxonomic lineage using the taxonomic identifiers provided by UniRef100 and the NCBI taxonomy. Eukaryotic proteins were excluded if the taxonomic lineage included the keywords "unclassified eukaryotes", "environmental samples", or if the base taxonomic label was at a broader taxonomic rank than species. Eukaryotic marker genes were identified and extracted if they aligned with Diamond to the 255 eukaryotic Benchmarking Universal Single-Copy Orthologs (BUSCO) marker genes (version 5) [25] with an alignment matching or exceeding the BUSCO cutoffs for sequence length and bit score.

\section{Training the taxonomic classifiers}

The classifiers of taxaTarget are a set of region-specific functions that use thresholds for family, genus, and species, to classify metagenomic reads. The thresholds for species, genus, and family $\left(S_{(M, W)}, G_{(M, W)}\right.$, and $F_{(M, W)}$, respectively) are learned for each non-overlapping 20 amino acid window, $W$, of each marker 
gene, $M$, in the taxaTarget database via training. As an example, the notation for the species threshold of the 140 to 160 amino acid window of marker gene UniRef100_COH4X5 (its UniRef100 identifier) would be $S_{\text {(UniRef10O_COH4X5, 140) }}$. The thresholds learned during training for each window, provide the basis for the taxaTarget classifiers.

To provide an overview, the training process began by decomposing the UniRef100 database into a set of reads. Each read was aligned to the taxaTarget database, yielding one or more alignment scores depending on how many marker genes it aligned to. Per alignment, each read was assigned a label, either "species", "genus", "family", or "negative", depending on its taxonomic relationship to the marker gene. A read that aligned to a marker gene from a broader rank than family was considered a negative. The set of labelled reads that mapped within the window of a marker gene were used to determine the thresholds for that window.

The alignment score used for training and classification was the mean bit score-the bit score of a read alignment divided by the alignment length. The mean bit score was used because there is an approximately linear relationship between the alignment bit score and alignment length [28], i.e., reads of different lengths, sequenced from the same gene, will align back to the marker gene with different bit scores, but similar mean bit scores. Therefore, training on the mean bit score allows for standardized classification of reads of different lengths.

In detail, training began by aligning all UniRef100 reads (reads are all 70 amino acid long k-mers, with step size 10, extracted from UniRef100), to the marker genes using Diamond BLASTP. The procedure for identifying the species, genus, and family thresholds $\left(S_{(M, W)}, G_{(M, W)}\right.$, and $F_{(M, W)}$, respectively) for each window, $W$, of each marker gene, $M$, in the taxaTarget database is as follows. To provide a specific example for ease of understanding, let us say we are trying to learn $S_{(M, W)}, G_{(M, W)}$, and $F_{(M, W)}$ for the 140

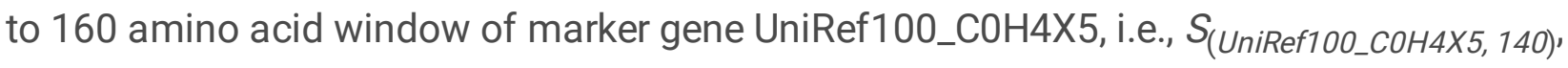

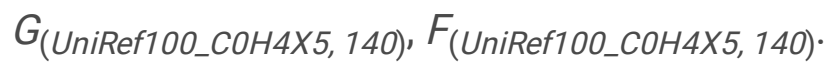

1) If a read alignment to UniRef100_COH4X5 started or ended within the 140 to 160 amino acid window (extending towards the end of the gene) with a minimum bit score of 60 and alignment length of at least 30 amino acids,

a) The mean bit score of the read alignment was extracted

b) A taxonomic label ("species", "genus", "family", or "negative") was assigned depending on the taxonomic relationship of the read to the marker gene (Plasmodium falciparum for UniRef100_COH4X5)

2) $S_{(\text {UniRef100_COH4X5, 140) }}, G_{(\text {UniRef100_COH4X5, 140) }}, F_{(\text {UniRef100_COH4X5, 140) }}$ were set to the minimum mean bit score of the reads mapped and corresponding to that taxonomic rank

3) Thresholds were adjusted or removed based on the following conditions 
a) A threshold was removed if there was no training data corresponding to its taxonomic rank

b) If the mean bit score of a broader taxonomic rank was higher than the threshold of a more specific taxonomic rank, that threshold was either adjusted to that mean bit score or removed if the mean bit score was higher than the threshold of the next most specific rank

c) The window was only kept in taxaTarget for classification if,

i) $S_{(\text {UniRef100_COH4X5, 140) }}$ had a value (in some cases reads did not map to a window)

ii) $S_{(\text {UniRef10O_COH4X5, 140) }}>\left\{G_{(\text {UniRef100_COH4X5, 140) }}, F_{(\text {UniRef100_COH4X5, 140) }}, \max (\right.$ negatives $\left.)\right\}$

4) The process was repeated for every window of every marker gene in the database.

At the end of the training process, the values for $S_{(M, W)}, G_{(M, W)}$, and $F_{(M, W)}$ were defined for each window, for each marker gene, subject to the filtering parameters described above. This provided the basis for the classifiers used by taxaTarget for classification.

\section{Taxonomic classification of metagenomic reads}

\section{Classifying individual reads}

Taxonomic classification with taxaTarget begins by aligning metagenomic reads to the taxaTarget database with Diamond BLASTX, yielding alignment scores. The highest mean bit score alignment(s) of individual reads are used for classification. If a read aligns with $100 \%$ identity to multiple marker genes from different species, the read is classified at the lowest common ancestor of the respective hits. Otherwise, individual reads are classified as follows:

1) The alignment of a read to a marker gene must be at least 30 amino acids long (gaps are counted)

2) A read alignment must either start or end in the window of a classifier and extend toward the end of the marker gene. If a read alignment overlaps multiple windows, the window used for classification is window $=$ floor $(\min ($ start, end $) / 20)$. If a classifier does not exist for that window, the read is not classified.

3) The classifier for a window consists of a function that uses the learned classification thresholds for species, genus, and family, $S_{(M, W)}, G_{(M, W)}$, and $F_{(M, W)}$, respectively, where $M$ is the specific marker gene the read aligned to and $W$ is the window.

4) The mean bit score of the read alignment is provided as input to the classifier

5) Classification 

a. If the mean bit score $\geq S_{(M, W)}$, classify the read at the species level
b. If the mean bit score $\geq G_{(M, W)}$, classify the read at the genus level
c. If the mean bit score $\geq F_{(M, W)}$, classify the read at the family level
d. Else, the read is not classified

\section{Generating the taxonomic profile of the metagenomic sample}

Once the individual reads have been taxonomically classified, taxaTarget uses several functions to analyze the pattern of mapped reads for each taxon and to filter out false positives from the taxonomic profile. The sources of false positives were identified through experiments or reported in the literature previously [22]. Each parameter has a default setting that can also be set by the user.

1. Thresholds determined from training data that lacked examples from broader taxonomic ranks or negatives are frequently overly permissive leading to false positive classifications. To address this factor, taxaTarget relies on "padding," a user tunable parameter, to adjust the thresholds higher (requiring a higher mean bit score in order to classify a sequence). The default padding added ( 0.5 by default) was determined via parameter sweep analyses detailed later in the Methods. The following padding strategy is used:

a. By default, $\mathrm{T}_{\text {padding }}=\mathrm{T}+0.5(\mathrm{I}-\mathrm{T})$, where $\mathrm{T}$ is the threshold (options are $S_{(M, W)}, G_{(M, W)}$, and $F_{(M,}$ $w_{\text {) }}$ ) before padding, $\mathrm{I}$ is the mean bit score of the identity alignment for the window, and $T_{\text {padding }}$ is the threshold value after padding is added

b. Padding is added using the following series of rules. Once a rule is met, the subsequent rules are not applied. During training, if no reads aligned to a marker gene,

i. At a rank broader than family, then padding is added to $S_{(M, W)}, G_{(M, W)}$, and $F_{(M, W)}$

ii. At a rank broader than genus, then padding is added to $S_{(M, W)}$ and $G_{(M, W)}$

iii. At a rank broader than species, then padding is added to $S_{(M, W)}$

2. As reported in [22] and supported by our observations, false positives frequently entail just one or two reads mapping to a single marker gene of an organism. To avoid such errors, taxaTarget requires that at least 3 reads be classified per taxonomic unit, and that reads map to at least 3 marker genes within a taxonomic unit for it to be reported in the output.

3. If sequencing samples the genomes in a metagenome randomly, then the reads should cover a genome, approximate, uniformly. False positives are often characterized by many reads mapping to a small subset of the marker genes from a particular taxon, perhaps due to a conserved domain. taxaTarget relies on outlier detection to filter out marker genes that have more mapped reads than 
expected for each taxon. The cutoff for the maximum expected number of mapped reads for a marker gene is approximated as follows:

a. Let $X=\left\{x_{1}, x_{2}, \ldots, x_{N}\right\}$, where $X$ is a vector of counts and $x_{i}$ is a single draw, sampled with replacement, from the set of marker genes, $\left\{m_{1}, m_{2}, \ldots, m_{K}\right\}$, where $N$ is the total number of reads mapped to the marker genes, and $\mathrm{K}$ is the total number of marker genes for a taxon. $C=$ $\operatorname{mode}(X) \times 2$.

b. Any marker gene with more mapped reads than the mean of $C$ after 100 experiments is excluded for the taxon being considered.

4. A source of error, also identified in [22], is that more species of a genus get detected than are present in the sample. Sometimes, this is due to the species lacking a complete set of marker genes in the taxaTarget database. To address such situations, taxaTarget examines the pattern of read mappings to the marker genes from the equivalent species using an extension of the strategy reported in [22]. First, a primary species is defined as the species with the most mapped reads that passes all previous filters. The primary species is assumed present, and the other species are compared to it to determine if they are present as well or false positives. For demonstration purposes, let us consider two scenarios (that taxaTarget handles) where two species, A and B, within the same genus have mapped reads, where $A$ is the primary species.

a. If the reads of species A and B map to non-overlapping sets of marker genes, the read counts are summed and the taxonomic profile reports both species as a single result indicating there is not enough information to determine whether either or both are present.

b. If the reads of species $A$ and $B$ map to partially overlapping sets of marker genes, species $B$ is considered a false positive if the ratio of reads mapped (normalized by the number of marker genes) to shared genes versus not-shared genes is less than 1 , with a tolerance of $10 \%$.

\section{Simulated data sets}

Four simulated datasets were created using ART [33] to generate single-end, Illumina HiSeq, 150 bp reads at $1 \mathrm{X}$ depth of coverage of the genomes described in Table 1. The four datasets were named nonmicroeukaryote, microeukaryote_in_DB, microeukaryote_not_in_DB, and sensitivity_analysis.

For the non-microeukaryote dataset, the GenBank assembly report (available at https://ftp.ncbi.nlm.nih.gov/genomes/ASSEMBLY_REPORTS/assembly_summary_genbank.txt) was parsed with a custom Python script to download one assembly per genus for each of the following phylogroups: metazoa (animals), embryophyta (plants), archaea, bacteria, and viruses. Per genus, the assemblies were first prioritized by assembly level, i.e., complete genome, chromosome, scaffold, contig. Then an assembly was randomly selected from the highest assembly level available for that genus. For analysis, simulated reads were combined into metagenomes by phylogroup and randomly down sampled 
to approximately 10 million reads. All reads simulated from the archaea and virus assemblies were used as they were already fewer than 10 million.

The assemblies for the microeukaryote_in_DB dataset were downloaded from the NCBI assembly database after searching for "eukaryota" and filtering for "Latest RefSeq", "Protists", "Fungi", "Complete genome", "Chromosome", "Representative." Here, we only used high quality assemblies to reduce the likelihood of contaminating sequences in the assemblies which could affect the classification results. The assemblies were further filtered to only include species that had sequences in the databases of each tool tested (taxaTarget, EukDetect and Metaxa2). The read sets were analyzed individually as well as a metagenome mixture where all the reads had been combined into a single fastq file.

The assemblies for the microeukaryote_not_in_DB were downloaded using the GenBank assembly report and a custom Python script that found species without sequences in any of the databases used by Metaxa2, EukDetect, and taxaTarget, but that did have sequences from the same genus in these databases. Each read set was analyzed individually.

The assemblies of two protist (Phytophthora sojae and Plasmodium falciparum) and one fungal (Aspergillus niger) species were downloaded from GenBank (GCA_000149755.2, GCA_000002765.2, GCA_000002855.2, respectively) to create the sensitivity_analysis dataset. Each species was represented in the database of each tool tested. Six read sets were created from each assembly, composed of 1,10 , $100,1 \times 10^{3}, 1 \times 10^{4}$, and $1 \times 10^{5}$ reads using a custom Python script to randomly select from the ART simulated reads. Each read set was analyzed individually.

\section{Parameter sweep analysis for adjusting classification thresholds}

As mentioned earlier, thresholds learned from training data that lacked examples from broader taxonomic ranks or negatives frequently allowed false positive classifications. To address this, taxaTarget relies on "padding" these thresholds, a user tunable parameter, to adjust the thresholds higher. The default amount of padding to add to thresholds was assessed via a parameter sweep analysis. The values tested for padding included $(0.0,0.1,0.2, \ldots, 1.0)$. The experiment involved three simulated datasets which were useful for assessing different performance metrics of taxaTarget.

First, the non-microeukaryote dataset was used to assess the false positive rate of taxaTarget-both with and without applying outlier detection (see above). The false positive rate was calculated as the proportion of reads from non-protist/non-fungi phylogroups (i.e., animals, archaea, bacteria, plants and viruses) that were misclassified as a protist or fungus.

Second, the metagenome of the microeukaryote_in_DB dataset was used to assess the relationship between the precision and the sensitivity of taxaTarget for species with sequences in the database. The precision was calculated using the number of true positive and false positive reads classified at the 
species level. The sensitivity of taxaTarget was calculated using the number of true positive and false negative species detected in the metagenome.

Third, the microeukaryote_not_in_DB dataset was used to assess the relationship between the precision and sensitivity of taxaTarget for species with no sequences in the database, but that have relatives from the same genus within the database. The precision and sensitivity were calculated as before except at the genus level.

\section{Limit of detection analysis using simulated data}

The sensitivity_analysis dataset (Table 1) was created to test the limit of detection of the tools for two protist (Plasmodium falciparum and Phytophthora sojae) and one fungal (Aspergillus niger) species across a range of depth of coverage of the genomes. These datasets were meant to mimic metagenomic samples where protist and fungal species occur with varying levels of sparse genomic coverage. The 3 species have genomes ranging in size from $2.3 \times 10^{7}$ bp to $8.3 \times 10^{7} \mathrm{bp}$. Every tool tested had reference sequences from these species in their database. Each read set was classified separately with the tools and the number of reads correctly classified at the genus level were recorded.

\section{Testing the false positive rate of the tools for non- protist/non-fungi species}

The non-microeukaryote dataset (Table 1) was used to test the false positive rate of the tools. The false positive rate was calculated as the proportion of reads from non-protist/non-fungi phylogroups (i.e., animals, archaea, bacteria, plants and viruses) that were misclassified as a protist or fungi. The mean false positive rate for all phylogroups was normalized by 10 million to derive the mean number of false positive reads per 10 million reads classified.

\section{Measuring the precision and sensitivity of the tools for protist and fungal species}

The microeukaryote_in_DB dataset (Table 1) was used to measure the precision of the tools when classifying protist and fungal species with reference sequences in the database. The tools were first applied to each read set individually to provide a baseline for the precision of the tools and then to the combined read set to assess the precision of the taxonomic profile when multiple species were present (sometimes multiple species from the same genus). The precision was calculated separately at the taxonomic levels of family, genus and species. The sensitivity of the tools was defined at the family, genus and species levels as the proportion of the total number species in the dataset where at least one read was classified correctly at the respective taxonomic levels. 
The microeukaryote_not_in_DB dataset (Table 1) was used to measure the precision of the tools when classifying protist and fungal species with no reference sequences (although there were reference sequences from other species within the corresponding genera) for the species in the databases used by the tools. The precision and sensitivity of the tools were measured as mentioned in the previous paragraph except only at the taxonomic levels of family and genus as the respective species are not represented in the reference collection.

\section{Analysis of real metagenomic data}

The accuracy of the tools for detecting known protist species in a microbiome was quantified using 57 Cameroonian shotgun metagenomic gut samples [34]. Polymerase chain reaction (PCR) had previously been performed on the samples to detect Blastocystis and microscopy to detect Entamoeba. Additionally, the previous study had identified several target protist species by mapping the metagenomic reads to a database of their genomes or 18S rRNA sequences [34]. We summarized and compared the taxonomic profiles of each tool with the findings of the original study. For Blastocystis, the PCR results were used as a "gold standard" for measuring the accuracy of the tools for detecting this parasite.

\section{Results}

\section{Database creation}

The reference database of taxaTarget is composed of orthologs of the 255 BUSCO eukaryotic marker genes. These marker genes tend to have a strong eukaryotic evolutionary signal and are less likely to generate false positives when used for taxonomic classification [18, 25]. Of these, 877,724 were identified as homologs of the 255 eukaryotic BUSCO marker genes. These sequences originate from 1,319 families, 2,564 genera and 4,232 species. As seen in Figure 2, many species had few marker genes (e.g., 1,152 species only had one marker gene identified); however, 2,619 species had over 50 marker genes and the median number of marker genes per species was 152 .

\section{Statistics about the trained classifiers}

Classifiers were trained for all non-overlapping 20 amino acid regions $\left(22.7 \times 10^{7}\right.$ potential regions) per protein in the database. About $\otimes$ of the regions $\left(7.4 \times 10^{6}\right)$ were excluded after training because the highest mean bit score alignments for family, genus, and species did not follow the pattern of species > genus > family (Figure 3). For the remaining $\square$ of regions, training data was often sparse i.e., $22.7 \%, 64.2 \%$, and $68.1 \%$ of the regions lacked negative-labeled, family-labeled, or genus-labeled data, respectively. The number of region-specific classifiers varied by orders of magnitude between marker genes, from $2.8 \times 10^{3}$ to $1.5 \times 10^{6}$ with a median of $4.3 \times 10^{4}$. There was also great variability per species, from 1 (e.g., Phytophthora andina) to $1.6 \times 10^{5}$ (e.g., Aureobasidium pullulans) with a median of $2.1 \times 10^{3}$ region- 
specific classifiers per species. Furthermore, there were cases where the mean bit score showed a high variability across windows of a marker gene within a single rank; the maximum mean bit score range observed was 1.94 across the windows of the Brix domain-containing protein of Hortaea werneckii (A0A3M7GIU8).

\section{Parameter sweep analysis: Padding thresholds trained with sparse data}

The first parameter sweep analysis used the non-microeukaryote metagenome to assess how the padding of thresholds, with and without outlier detection, affected the number of non-microeukaryote reads falsely classified as microeukaryotic (protist or fungal) species (Figure 4A). Here, outlier detection refers to whether taxaTarget is set to remove marker genes with more reads mapped than expected (see Methods).

As seen in Figure 4A, irrespective of padding, taxaTarget always performed better when using outlier detection, especially at low threshold padding, i.e., 0.2 or less. Without outlier detection, the number of false positive reads per 10 million reads classified decreased from 179 to 7 as the threshold padding increased from 0 to 1 . With outlier detection, the same value over the same range decreased from 26 to 4 . From 0.1 to 0.2 padding there was an unexpected increase in the number of false positive reads classified as embryophyta. This was caused by several species where the pattern of mapped reads to marker genes was not uniformly distributed at 0.1 padding-resulting in them being filtered out of the taxonomic profile by taxaTarget. However, at 0.2 padding the number of reads mapped to marker genes was greatly reduced and approximated uniform coverage-resulting in them being wrongly included in the taxonomic profile. The phylogroups that contributed to the most false positives, irrespective of padding level, were the bacteria and embryophyta. For bacteria, misclassified reads were most frequently classified as fungi, chlorophyta, and choanoflagellata. For example, at 0.5 padding there were 15 misclassified reads, of which 7, 5, and 4 were assigned to fungi, chlorophyta, and euglenozoa, respectively. For embryophyta, misclassified reads were most frequently classified as chlorophyta and charophyceae-green algae. For example, at 0.5 padding there were 23 misclassified reads, 20 were assigned to chlorophyta (the other 3 were assigned to stramenopiles). The viruses, archaea, and metazoa did not produce false positives, only did so at a low rate (irrespective of threshold padding), or only at a padding of 0.1 or lower, respectively.

The second analysis used the microeukaryote_in_DB metagenome to assess the effect of padding thresholds on the precision and sensitivity of taxaTarget at the species level for species with sequences in the reference database (Figure 4B). The sensitivity was 1.0 regardless of padding, i.e., all species were detected at the species level in the metagenome. With respect to precision, as padding increased from 0 to 1 there was a slight increase from 0.951 to 0.981 , respectively. 
The third analysis used the microeukaryote_not_in_DB dataset to assess the effect of padding thresholds on the precision and sensitivity of taxaTarget for species without sequences in the database (Figure 4C). The precision was assessed at the genus level. Similarly, the sensitivity was calculated as the proportion of species detected at the genus level. Overall, the sensitivity was 0.952 from 0 to 0.8 padding and 0.94 thereafter. The precision continuously increased from 0.814 to 0.916 from a padding of 0 to 1 , respectively.

Together, the parameter sweep analyses led us to choose a threshold padding of 0.5 as the default setting for taxaTarget, but users can adjust this parameter.

\section{Limit of detection analysis}

The limit of detection for each tool was tested by classifying read sets simulated from genomes, where the number of reads varied by 6 orders of magnitude $\left(1,10,100,1 \times 10^{3}, 1 \times 10^{4}, 1 \times 10^{5}\right)$ (Figure 5). The datasets were simulated from the genomes of two protist species (Plasmodium falciparum and Phytophthora sojae) and one fungal species (Aspergillus niger). The genomes ranged in size from 23, 34, and $83 \mathrm{Mbp}$ for Plasmodium falciparum, Aspergillus niger and Phytophthora sojae, respectively. Each species had reference sequences in the database of each tool. The sensitivity of a tool was measured as the number of reads correctly classified at the genus level.

Amongst the tools, taxaTarget showed the greatest sensitivity, only requiring 100 reads to detect Aspergillus and 1,000 reads to detect Phytophthora and Plasmodium. EukDetect required 10,000 reads to detect Aspergillus and Phytophthora and 100,000 reads to detect Plasmodium. Metaxa2 showed the least sensitivity, unable to detect Phytophthora given a million reads, detecting Aspergillus given 100,000 reads, and 10,000 reads to detect Plasmodium.

\section{False positive rate when classifying simulated metagenomes composed of non-fungi/non-protist phylogroups}

Microbiomes can be composed of hundreds or thousands of microbial species-usually only a small proportion are microeukaryotes (mostly protists and fungi) [17,35]. The non-microeukaryote dataset was used to quantify the false positive rate of the tools when classifying non-protist/non-fungal phylogroups.

As seen in Table 2, EukDetect had the lowest overall false positive rate of zero. The mean number of false positive reads per 10 million reads classified for Metaxa2 and taxaTarget was 4.4 and 7.6, respectively. For taxaTarget, the false positive rate was mostly driven by bacteria identified as Choanoflagellata and plants identified as green algae (Charophyceae and Chlorellales). 


\section{Classification performance for known fungal and protist species}

Here we assess the precision of the tools when applied to data derived from genomes found in the databases of all tools.

The precision of the tools was tested under two conditions. First, classifying read sets simulated from individual genomes (Table 3). Second, classifying the metagenome of the combined read sets (Table 4). The first condition helped establish a baseline of precision for each tool when only a single species was present. The second condition tested the precision of the taxonomic profile of the tools when there were many species in a sample, sometimes multiple from the same genus (24 of the 54 species came from a genus with multiple species).

When classifying read sets simulated from individual genomes, all tools showed high precision (>95\%) at genus and species levels, but only taxaTarget had a sensitivity of $100 \%$, i.e., detected all species (Table 3). In contrast, EukDetect had a sensitivity of $90.7 \%$, misclassifying 5 species as other species. Metaxa2 had the lowest sensitivity, only detecting $31.5 \%$ of the species and classifying 160 reads in total at the species level. In terms of precision, taxaTarget and Metaxa2 were the best performing at the species level, 97.3\% and $97.4 \%$, respectively, with EukDetect slightly lower at $95.3 \%$.

When classifying the simulated reads combined into a single metagenome, all tools still showed high precision ( $\geq 95 \%$ ). Metaxa2 and taxaTarget still had the highest precision at the species level, $97.0 \%$ and $97.3 \%$, respectively, and taxaTarget still had a sensitivity of $100 \%$ (Table 4). In contrast, EukDetect showed a substantial loss in sensitivity (only detecting one species per genus when multiple species were present), detecting $68.5 \%$ of the species, in contrast to $90 \%$ when data from each species is provided separately. Further, Metaxa2 detected the same number of species as previously but with even fewer classified reads, 130 in total.

\section{Classification performance for unknown fungal and protist species}

We sought to test whether the tools could detect species that were not represented in their databases, but that had reference sequences from the same genus. We tested the tools using the microeukaryote_not_in_DB dataset consisting of genomic read sets simulated at $1 \mathrm{X}$ coverage for 85 protist/fungal species that had no reference sequences in the databases of any tool, but that had sequences from species related at the genus level (Table 5).

In terms of sensitivity, taxaTarget detected the most species in the sample at the family and genus levels, $97.6 \%$ and $95.2 \%$, respectively. Meanwhile, EukDetect detected about $50 \%$ of the species at the family and genus levels, and Metaxa2 detected $28 \%$ or fewer. In terms of precision, all tools detected the species at 
the family level with $\geq 90 \%$. However, EukDetect was the most precise in detecting the species at family and genus levels with $99.9 \%$ and $90.4 \%$ precision, respectively. taxaTarget showed slightly lower precision, detecting species at the family and genus levels with $94.5 \%$ and $89.1 \%$ precision, respectively. In contrast, Metaxa2 had a precision of only $41.6 \%$ for detecting species at the genus level.

\section{Detection of target species in the Cameroonian gut metagenomes}

The accuracy of the tools for detecting known protist species in a microbiome was quantified using 57 Cameroonian shotgun metagenomic gut samples (Table 6). PCR had previously been performed on the samples to detect Blastocystis and microscopy to detect Entamoeba. Additionally, several target protist species had been detected by mapping the metagenomic reads to a database of their genomes or to their 18S rRNA [34].

For Blastocystis, the PCR results were used as the "gold standard" for the analysis. The accuracy of the tools was assessed at the genus level because the databases of the tools did not contain all the Blastocystis species detected by PCR. Metaxa2 had the highest agreement with the PCR results, $96.5 \%$, with taxaTarget and EukDetect showing slightly less agreement with the PCR results, $89.5 \%$ and $82.5 \%$, respectively. All the tools detected Blastocystis in one sample that was PCR negative. For the 5 PCR positive samples where taxaTarget did not detect Blastocystis, three samples only had one or two classified reads-taxa with fewer than 3 classified reads are removed from the taxonomic profile by taxaTarget. The other two samples had three and seven reads, respectively, but they mapped to fewer than three marker genes, the minimum number required by taxaTarget to be included in the taxonomic profile.

Previously, microscopy of the Cameroon samples had been found less sensitive for Entamoeba than mapping the metagenomic reads to reference genomes-19 and 36 samples were positive using microscopy and read mapping, respectively [34]. For our analysis, Metaxa2 and taxaTarget were the most sensitive tools, detecting Entamoeba in 21 and 13 samples, respectively. All Entamoeba positive samples had also been found positive for Entamoeba by the previous study. However, only 67\% (Metaxa2) and 69\% (taxaTarget) of the Entamoeba positive samples agreed with the microscopy results. In contrast, EukDetect did not detect Entamoeba in any samples.

The previous analysis had also detected Endolimax, Enteromonas, and Giardia [34]. None of the tools detected the genus Endolimax and Metaxa2 and EukDetect did not detect Enteromonas or Giardia. In contrast, taxaTarget detected either Giardia or Hexamitidae (the family of Giardia) in the two samples where it was previously identified. Additionally, despite not having any Enteromonas sequences in the database, taxaTarget detected Hexamitidae (the family of Enteromonas) in 3 of 4 samples where Enteromonas had been identified previously. 


\section{Comparison of the taxonomic profiles for the 57 Cameroon gut samples}

The total diversity of microeukaryotes was compared for the three tools (Figure 6). EukDetect, taxaTarget, and Metaxa2 detected 12, 20, and 22 microeukaryote genera, respectively. Amongst these genera, only Blastocystis was detected by all-was also the most frequently identified. Amongst the tools, taxaTarget had the greatest overlap with the other tools, sharing 9 genera with EukDetect and 3 with Metaxa2. The three additional genera that EukDetect identified, but not taxaTarget, were either not in the database of taxaTarget (Kodamaea), were not detected and would not have been reported in the results for having too few marker genes (i.e., 3) in the database (Volvariella), or were detected at too low of abundance and were filtered out of the taxonomic profile (Bactrocera). Similarly, of the 18 genera uniquely detected by Metaxa2, ten were not in the database of taxaTarget, two had three or fewer marker genes in the database, and four genera either had fewer than three mapped reads per sample or the reads mapped to a single marker gene. Further, although Aplanochytrium was not detected at the genus level, its family was detected. Only the genus Amphora showed no signs of detection at the family or genus levels despite having sufficient marker genes in the taxaTarget database to be reported in the taxonomic report.

Consistent with other studies of the human gut eukaryome [35], no genus was found in all samples and most genera were rare. For perspective, there were only three and five genera identified in five or more samples by EukDetect and Metaxa2, respectively, and five for taxaTarget. For these genera, taxaTarget contained all detected by EukDetect and all but one by Metaxa2, Korotnevella, which is not in the taxaTarget database.

Of the 20 genera detected by taxaTarget in the 57 Cameroonian gut samples, 16 had been observed in the human gut microbiome before (Table 8). Of the remaining four, Chaetoceros, Perkinsus, and Wallemia potentially could have been transiently introduced to the gut microbiome if a person consumed foods that carried them. Amongst the genera identified by taxaTarget were parasites with clinical relevance to humans such as Trichinella, Schistosoma, Strongyloides, Digenea, Necator, Trypanosoma, and Giardia.

\section{Computational resource usage of the tools}

The runtime and maximum random access memory (RAM) usage (Figure 7) of the tools were recorded for the non-microeukaryote and microeukaryote_in_DB simulated metagenomic datasets as well as one of the Cameroonian gut metagenomes-a total of $\sim 91$ million reads. All tools were run using 12 CPUs on a 256 gigabytes RAM node (Intel $\circledast$ Xeon $\circledast$ central processing unit, E5-2650, version 3, at 2.3 gigahertz). In terms of computational efficiency, taxaTarget and EukDetect had the shortest total runtime, 102 minutes for both, and the smallest maximum memory requirement (2 gigabytes and 1.7 gigabytes, respectively). In contrast, although Metaxa2 did not have a substantially longer total runtime, 130 minutes, its memory usage ranged widely from a minimum of 46 megabytes to a maximum of 12 gigabytes depending on the dataset being analyzed. 


\section{Discussion}

Metagenomics is a powerful tool for exploring the complex microbial communities of bacteria, archaea, viruses, and eukaryotes that compose microbiomes. However, despite the many tools available, we are still not able to comprehensively assess the taxonomic composition of metagenomes. Here we report an important step forward for the identification of microeukaryotic species in metagenomic data. taxaTarget is a fast and computationally efficient supervised learning method that learned region-specific classification thresholds from the UniRef 100 database. Our results show this approach can match and often outperform other state-of-the-art, marker-gene-based tools in terms of precision and sensitivity. These gains applied for species with sequences in the database and for species which only have close relatives in the database-extending the predictive power of the scarce sequence data available for microeukaryotes. Although the use of a protein database for taxaTarget could be expected to have high sensitivity for divergent sequences, due to the redundancy of the genetic code, it was not expected to be more precise than tools using nucleotide databases (and thus might be even more precise with a nucleotide database).

Perhaps the greatest challenge for developing taxaTarget was the scarcity of reference sequence data versus their estimated species diversity [36]. It is imperative that more genomes/transcriptomes/proteomes are sequenced for eukaryotic species to increase the breadth of detectable taxa in metagenomes. As seen in the analysis of the Cameroonian gut metagenomes, although taxaTarget identified more microeukaryotes than the previous study of the dataset, including clinically relevant parasites, the biased representation of species in the databases of the tools acutely affected the results. EukDetect and taxaTarget-which use a similar marker gene database-detected an almost non-overlapping set of genera compared to Metaxa2, which uses a custom database of small and large ribosomal subunits as well as COI genes. For reference, the small ribosomal subunit 18S rRNA database in Metaxa2 (which includes SILVA and PR2) currently has nearly an order of magnitude more species represented than in protein or genome databases. As a side, we did not include the 18S rRNA sequences in taxaTarget because the additional species would only have had one marker sequence, which is insufficient for handling classification errors with our current approach.

Scarce data is also problematic for supervised learning because the accuracy of classification is often dependent upon the number and diversity of labelled training data. For taxaTarget, the classification thresholds of many regions were learned using five or fewer data points, preventing the use of more sophisticated machine learning approaches. Some regions lacked some or all the thresholds because there was no training data. Together, this prevented us from using neighboring windows to impute missing thresholds. However, as more data becomes available, future work should test imputation methods (a common solution for handling missing data in supervised learning [37]) as they might increase sensitivity/precision by including more regions for classification.

The main solution, in taxaTarget, for handling classification errors due to missing data was to pad thresholds that lacked training data at broader taxonomic ranks, an approach that was highly effective 
for reducing the number of classification errors. However, even at high threshold padding, false positives from non-microeukaryotes persisted, highlighting the need for more comprehensive training data. One persistent source of classification errors were pileups of false positive reads from non-microeukaryotes in specific regions of specific marker genes. Provided sufficient depth of coverage, these events might be simply filtered using sequencing coverage statistics [38]; however, it was common to observe 100 or fewer mapped reads per microeukaryotic taxa in the fecal metagenomes we analyzed, which is far less than $1 \mathrm{X}$ depth of coverage. The simple and effective solution developed for taxaTarget used a variant of the "urn problem" to detect and remove outlier marker genes. The marker genes of a taxa were treated like marbles with different colors in an urn and the number of reads mapped to the marker genes were treated as draws, with replacement, from the urn. Averaged over 100 simulations, the count of the marker gene drawn most often provided a useful baseline for the highest expected variant.

Another source of error, related to missing data, (observed here and previously [22]) was caused by closely related species with partially overlapping gene sets in the database. For example, if the marker genes of species $A$ are a subset of those found in species B, both from the same genus, there is the chance that some metagenomic reads sequenced from A will get classified as B. EukDetect partially addressed this problem by filtering out species where more reads mapped to unshared versus shared marker genes with another species in the same genus. taxaTarget implemented a modified version of this solution and found it effective, although some errors persisted. EukDetect applied an additional filter (taxaTarget does not use), to further address this source of error, which removes species if the global percent identity of aligned reads across genes shared with the primary species is less than that for the primary species. It was acknowledged in [22] that this might result in only detecting the primary species of a genus, but this bias was observed for EukDetect even when using a simulated metagenome where each species had $1 \mathrm{X}$ depth of coverage. Future work should explore if statistical methods for assessing ambiguity in taxonomic classification, like ATLAS [39], can be adapted for the classification of microeukaryotes in metagenomic data (ATLAS in its current form was not usable because it depends on a database with good species representation of the metagenome being analyzed).

A major benefit of using a supervised learning approach was that training provided granular information about the discriminatory power of each region of the database sequences (something Metaxa2 and EukDetect do not provide), which sometimes varied widely for adjacent regions (this information is provided for download with taxaTarget and might be useful for the study of sequence evolution). Where EukDetect removed 41 of the 255 eukaryotic BUSCO marker gene families from its database for regions conserved in bacteria, taxaTarget was able to retain all 255 , by only excluding the regions that lacked discriminatory power.

Previously, the most expensive step for creating a tool like taxaTarget was the training process. To be able to classify metagenomic reads of variable length, MetaPhyler and ROCker were trained with a minimum of three read sets of different length $[28,29]$. However, we found that the longest read set was sufficient for training because the mean bit scores of the alignments were similar to those of the shorter read lengths. This resulted in a threefold reduction in training time. A potential future research area is 
exploring the replacement of the read mapping step of training with directly using the pairwise alignments of the sequences in the database.

Future work should test the methods of taxaTarget when extended to the marker genes of other phylogroups such as bacteria, archaea, and viruses. Furthermore, the methods should be tested when extended to entire sequence databases-which might improve sensitivity and specificity. Ideally, given sufficient data, training could identify which regions of a genome or proteome are useful for taxonomic classification. However, the use of sequences other than marker genes might not be feasible without the development of additional algorithms for handling missing data and the resulting classification errors. Lastly, despite highlighting the performance gains of data-driven methods, taxaTarget still implemented multiple user-defined thresholds, which also might be optimized or replaced with data-driven methods.

\section{Conclusions}

Our results show that the taxonomic classification of microeukaryotes in metagenomic data can be improved by utilizing data-driven methods that learn classification thresholds from the structure of an input database. These gains applied for species with sequences in the database and for species which only have close relatives in the database. The scarcity of available reference sequences still presents many challenges for the metagenomic taxonomic classification of microeukaryotes. More genomes/transcriptomes/proteomes need to be sequenced and methods for handling missing data and the resulting classification errors need to be further explored. The implementation of these methods will help facilitate a more taxonomically comprehensive analysis of metagenomic data and expand our knowledge about roles and diversity of eukaryotes in microbial communities.

\section{Declarations}

\section{Data availability}

The source code and a tutorial for installing and running taxaTarget can be found on GitHub at https://github.com/SethCommichaux/taxaTarget. The taxaTarget database can be downloaded from https://obj.umiacs.umd.edu/taxatarget/archive.tar.gz.

All data used for analysis in this study are publicly available. The Cameroonian gut metagenomes can be found under NCBI BioProject PRJEB27005. The NCBI Assembly accessions for the non-microeukaryote dataset, microeukaryote_in_DB, and microeukaryote_not_in_DB datasets can be found in the supplementary materials.

\section{Acknowledgements}

Not applicable 


\section{Funding}

MP was funded in part by the $\mathrm{NIH}$, award R01-Al-100947. KJ was supported by the University of Maryland, Joint Institute for Food Safety and Applied Nutrition (JIFSAN) through cooperative agreement \#5U01-FD001418, provided by the U.S. Food and Drug Administration, Center for Food Safety and Applied Nutrition (FDA, CFSAN).

\section{Competing interests}

The authors declare that they have no competing interests

\section{Author's contributions}

SC, AO, MP, and HR designed the study. SC, MP, HR, KJ, and HM designed the algorithms. SC analyzed the data and wrote the manuscript. SC, MP, HR, KJ, and AO interpreted the data. All authors read, provided feedback, and approved the final manuscript.

\section{Ethics approval and consent to participate}

Not applicable.

\section{Consent for publication}

Not applicable

\section{Rights and permissions}

Open source

\section{References}

1. Hawksworth DL, Lücking R: Fungal Diversity Revisited: 2.2 to 3.8 Million Species. Microbiol Spectr2017, 5(4).

2. Larsen BB, Miller EC, Rhodes MK, Wiens JJ: Inordinate Fondness Multiplied and Redistributed: the Number of Species on Earth and the New Pie of Life. The Quarterly Review of Biology2017, 92(3):229-265.

3. Pawlowski J, Audic S, Adl S, Bass D, Belbahri L, Berney C, Bowser SS, Cepicka I, Decelle J, Dunthorn Met al: CBOL Protist Working Group: Barcoding Eukaryotic Richness beyond the Animal, Plant, and 
Fungal Kingdoms. PLoS Biology2012, 10(11):e1001419.

4. Burki F, Roger AJ, Brown MW, Simpson AGB: The New Tree of Eukaryotes. Trends in Ecology \& Evolution2020, 35(1):43-55.

5. Rousseaux C, Gregg W: Interannual Variation in Phytoplankton Primary Production at A Global Scale. Remote Sensing2013, 6(1):1-19.

6. Wong Z-W, Faulder K, Robinson JL: Does Dientamoeba fragilis cause diarrhea? A systematic review. Parasitol Res2018, 117(4):971-980.

7. Deng L, Wojciech L, Gascoigne NRJ, Peng G, Tan KSW: New insights into the interactions between Blastocystis, the gut microbiota, and host immunity. PLoS Pathog2021, 17(2):e1009253.

8. Hirt RP: Mucosal microbial parasites/symbionts in health and disease: an integrative overview. Parasitology2019, 146(9):1109-1115.

9. Campo J, Bass D, Keeling PJ: The eukaryome: Diversity and role of microeukaryotic organisms associated with animal hosts. Functional Ecology2019.

10. Parfrey LW, Walters WA, Lauber CL, Clemente JC, Berg-Lyons D, Teiling C, Kodira C, Mohiuddin M, Brunelle J, Driscoll Met al: Communities of microbial eukaryotes in the mammalian gut within the context of environmental eukaryotic diversity. Front Microbio/2014, 5:298.

11. Even G, Lokmer A, Rodrigues J, Audebert C, Viscogliosi E, Ségurel L, Chabé M: Changes in the Human Gut Microbiota Associated With Colonization by sp. and spp. in Non-Industrialized Populations. Front Cell Infect Microbio/2021, 11:533528.

12. New FN, Brito IL: What Is Metagenomics Teaching Us, and What Is Missed? Annu Rev Microbio/2020, 74:117-135.

13. Laforest-Lapointe I, Arrieta M-C: Microbial Eukaryotes: a Missing Link in Gut Microbiome Studies. mSystems2018, 3(2).

14. Hooks KB, O'Malley MA: Contrasting Strategies: Human Eukaryotic Versus Bacterial Microbiome Research. J Eukaryot Microbio/2019.

15. Caron DA, Worden AZ, Countway PD, Demir E, Heidelberg KB: Protists are microbes too: a perspective. The ISME Journa/2009, 3(1):4-12.

16. Bik HM, Porazinska DL, Creer S, Caporaso JG, Knight R, Thomas WK: Sequencing our way towards understanding global eukaryotic biodiversity. Trends Ecol Evo/2012, 27(4):233-243.

17. Jacquiod S, Stenbæk J, Santos SS, Winding A, Sørensen SJ, Priemé A: Metagenomes provide valuable comparative information on soil microeukaryotes. Research in Microbiology2016, 167(5):436-450.

18. Saary P, Mitchell AL, Finn RD: Estimating the quality of eukaryotic genomes recovered from metagenomic analysis with EukCC. Genome Bio/2020, 21(1):244.

19. West PT, Probst AJ, Grigoriev IV, Thomas BC, Banfield JF: Genome-reconstruction for eukaryotes from complex natural microbial communities. Genome Res2018, 28(4):569-580. 
20. Lu J, Salzberg SL: Removing contaminants from databases of draft genomes. PLoS Comput Bio/2018, 14(6):e1006277.

21. Steinegger $M$, Salzberg SL: Terminating contamination: large-scale search identifies more than 2,000,000 contaminated entries in GenBank. Genome Bio/2020, 21(1):115.

22. Lind AL, Pollard KS: Accurate and sensitive detection of microbial eukaryotes from whole metagenome shotgun sequencing. Microbiome2021, 9(1):58.

23. Nousias $\mathrm{O}$, Montesanto F: Metagenomic profiling of host-associated bacteria from 8 datasets of the red alga Porphyra purpurea with MetaPhIAn3. Mar Genomics2021:100866.

24. Bengtsson-Palme J, Hartmann M, Eriksson KM, Pal C, Thorell K, Larsson DGJ, Nilsson RH: METAXA2: improved identification and taxonomic classification of small and large subunit rRNA in metagenomic data. Mol Ecol Resour2015, 15(6):1403-1414.

25. Simão FA, Waterhouse RM, loannidis P, Kriventseva EV, Zdobnov EM: BUSCO: assessing genome assembly and annotation completeness with single-copy orthologs. Bioinformatics2015, 31(19):3210-3212.

26. Wolf YI, Novichkov PS, Karev GP, Koonin EV, Lipman DJ: The universal distribution of evolutionary rates of genes and distinct characteristics of eukaryotic genes of different apparent ages. Proc Natl Acad Sci U S A2009, 106(18):7273-7280.

27. Marcelino VR, Clausen PTLC, Buchmann JP, Wille M, Iredell JR, Meyer W, Lund O, Sorrell TC, Holmes EC: CCMetagen: comprehensive and accurate identification of eukaryotes and prokaryotes in metagenomic data. Genome Bio/2020, 21(1):103.

28. Liu B, Gibbons T, Ghodsi M, Pop M: MetaPhyler: Taxonomic profiling for metagenomic sequences. 2010 IEEE International Conference on Bioinformatics and Biomedicine (BIBM)2010.

29. Orellana LH, Rodriguez-R LM, Konstantinidis KT: ROCker: accurate detection and quantification of target genes in short-read metagenomic data sets by modeling sliding-window bitscores. Nucleic Acids Res2017, 45(3):e14.

30. Menzel P, Ng KL, Krogh A: Fast and sensitive taxonomic classification for metagenomics with Kaiju. Nat Commun2016, 7:11257.

31. Buchfink B, Xie C, Huson DH: Fast and sensitive protein alignment using DIAMOND. Nature Methods2015, 12(1):59-60.

32. Consortium TU, The UniProt C: The Universal Protein Resource (UniProt). Nucleic Acids Research2007, 36(Database):D190-D195.

33. Huang W, Li L, Myers JR, Marth GT: ART: a next-generation sequencing read simulator. Bioinformatics2012, 28(4):593-594.

34. Lokmer A, Cian A, Froment A, Gantois N, Viscogliosi E, Chabé M, Ségurel L: Use of shotgun metagenomics for the identification of protozoa in the gut microbiota of healthy individuals from worldwide populations with various industrialization levels. PLOS ONE2019, 14(2):e0211139. 
35. Nash AK, Auchtung TA, Wong MC, Smith DP, Gesell JR, Ross MC, Stewart CJ, Metcalf GA, Muzny DM, Gibbs RAet al: The gut mycobiome of the Human Microbiome Project healthy cohort. Microbiome2017, 5(1):153.

36. Sibbald SJ, Archibald JM: More protist genomes needed. Nat Ecol Evo/2017, 1(5):145.

37. Kang $\mathrm{H}$ : The prevention and handling of the missing data. Korean Journal of Anesthesiology2013, 64(5):402.

38. Deng C, Daley T, Calabrese P, Ren J, Smith AD: Predicting the Number of Bases to Attain Sufficient Coverage in High-Throughput Sequencing Experiments. Journal of Computational Biology2019, 27(7):1130-1143.

39. Shah N, Meisel JS, Pop M: Embracing Ambiguity in the Taxonomic Classification of Microbiome Sequencing Data. Front Genet2019, 10:1022.

40. Nieves-Ramírez ME, Partida-Rodríguez O, Laforest-Lapointe I, Reynolds LA, Brown EM, Valdez-Salazar A, Morán-Silva P, Rojas-Velázquez L, Morien E, Parfrey LWet al: Asymptomatic Intestinal Colonization with Protist Blastocystis Is Strongly Associated with Distinct Microbiome Ecological Patterns. mSystems2018, 3(3).

41. Iyer LR, Verma AK, Paul J, Bhattacharya A: Phagocytosis of Gut Bacteria by Entamoeba histolytica. Frontiers in Cellular and Infection Microbiology2019, 9.

42. Spatz M, Richard ML: Overview of the Potential Role of Malassezia in Gut Health and Disease. Frontiers in Cellular and Infection Microbiology2020, 10.

43. Hallen-Adams HE, Suhr MJ: Fungi in the healthy human gastrointestinal tract. Virulence2017, 8(3):352-358.

44. Campa-Córdova Al, Luna-González A, Ascencio F, Cortés-Jacinto E, Cáceres-Martínez CJ: Effects of chloramphenicol, erythromycin, and furazolidone on growth of Isochrysis galbana and Chaetoceros gracilis. Aquaculture2006, 260(1-4):145-150.

45. Dimidi E, Cox SR, Rossi M, Whelan K: Fermented Foods: Definitions and Characteristics, Impact on the Gut Microbiota and Effects on Gastrointestinal Health and Disease. Nutrients2019, 11(8).

46. Afrin T, Murase K, Kounosu A, Hunt VL, Bligh M, Maeda Y, Hino A, Maruyama H, Tsai IJ, Kikuchi T: Sequential Changes in the Host Gut Microbiota During Infection With the Intestinal Parasitic Nematode Strongyloides venezuelensis. Frontiers in Cellular and Infection Microbiology2019, 9.

47. Santiago-Rodriguez TM, Fornaciari G, Luciani S, Dowd SE, Toranzos GA, Marota I, Cano RJ: Taxonomic and predicted metabolic profiles of the human gut microbiome in pre-Columbian mummies. FEMS Microbiol Ecol2016, 92(11).

48. Gouba N, Raoult D, Drancourt M: Eukaryote culturomics of the gut reveals new species. PLoS One2014, 9(9):e106994.

49. Berrilli F, Di Cave D, Cavallero S, D'Amelio S: Interactions between parasites and microbial communities in the human gut. Frontiers in Cellular and Infection Microbiology2012, 2. 
50. Zajc J, Gunde-Cimerman N: The Genus From Contamination of Food to Health Threat. Microorganisms2018, 6(2).

51. Wijayalath W, Majji S, Kleschenko Y, Pow-Sang L, Brumeanu TD, Villasante EF, Vasta GR, FernándezRobledo J-A, Casares S: Humanized HLA-DR4 Mice Fed with the Protozoan Pathogen of Oysters Perkinsus Marinus (Dermo) Do Not Develop Noticeable Pathology but Elicit Systemic Immunity. PLOS ONE2014, 9(1):e87435.

52. Dacks J: Faculty Opinions recommendation of On the reversibility of parasitism: adaptation to a freeliving lifestyle via gene acquisitions in the diplomonad Trepomonas sp. PC1. Faculty Opinions Post-Publication Peer Review of the Biomedical Literature2017.

53. Bucková B, Hurníková Z, Lauková A, Revajová V, Dvorožňáková E: The anti-parasitic effect of probiotic bacteria via limiting the fecundity of Trichinella spiralis female adults. Helminthologia2018, 55(2):102-111.

\section{Tables}

Table 1

The composition of the simulated metagenomic datasets

\begin{tabular}{|c|c|c|c|c|c|}
\hline Data set & Phylogroup & $\begin{array}{l}\text { Number } \\
\text { of } \\
\text { genera }\end{array}$ & $\begin{array}{l}\text { Number } \\
\text { of } \\
\text { species }\end{array}$ & $\begin{array}{l}\text { Number of } \\
\text { assemblies }\end{array}$ & $\begin{array}{l}\text { Number of } \\
\text { reads } \\
\text { simulated }\end{array}$ \\
\hline \multirow[t]{5}{*}{ non-microeukaryote } & Animals & 383 & 383 & 383 & $10,022,536$ \\
\hline & Archaea & 139 & 139 & 139 & $2,511,659$ \\
\hline & Bacteria & 2,761 & 2,761 & 2,761 & $10,653,348$ \\
\hline & Plants & 77 & 77 & 77 & $10,140,188$ \\
\hline & Viruses & 2,171 & 2,171 & 2,171 & 464,121 \\
\hline microeukaryote_in_DB & $\begin{array}{l}\text { Protists, Fungi } \\
\text { (species represented } \\
\text { in all tool databases) }\end{array}$ & 39 & 54 & 54 & $7,536,554$ \\
\hline microeuryote_not_in_DB & $\begin{array}{l}\text { Protists, Fungi } \\
\text { (species not } \\
\text { represented in } \\
\text { database of any tool) }\end{array}$ & 83 & 83 & 83 & $32,052,306$ \\
\hline \multirow[t]{3}{*}{ sensitivity_analysis } & $\begin{array}{l}\text { Plasmodium } \\
\text { falciparum }\end{array}$ & 1 & 1 & 1 & 155,278 \\
\hline & Phytophthora sojae & 1 & 1 & 1 & 528,057 \\
\hline & Aspergillus niger & 1 & 1 & 1 & 225,635 \\
\hline
\end{tabular}


Table 2

The false positive rate of the tools for the simulated metagenomes from non-protist/non-fungi phylogroups

\begin{tabular}{|lllllll|}
\hline Tool & Archaea & Bacteria & Plants & Animals & Viruses & $\begin{array}{l}\text { Mean number of false } \\
\text { positive reads per } 10 \text { million } \\
\text { reads classified }\end{array}$ \\
\hline EukDetect & 0 & 0 & 0 & 0 & 0 & 0 \\
Metaxa2 & 0 & $6.6 \times 10^{-7}$ & $5.9 \times 10^{-7}$ & $2.0 \times 10^{-7}$ & 0 & 4 \\
taxaTarget & $1.2 \times 10^{-6}$ & $1.5 \times 10^{-6}$ & $2.3 \times 10^{-6}$ & 0 & 0 & 8 \\
\hline
\end{tabular}

Table 3

Precision of tools for individually classifying simulated genomic ( $1 \mathrm{X}$ depth of coverage) data from 54 protist/fungal species with reference sequences in the database of each tool.

\begin{tabular}{|llllll|}
\hline Tool & $\begin{array}{l}\text { Precision } \\
\text { at family } \\
\text { level }(\%)\end{array}$ & $\begin{array}{l}\text { Precision } \\
\text { at genus } \\
\text { level }(\%)\end{array}$ & $\begin{array}{l}\text { Precision at } \\
\text { species } \\
\text { level }(\%)\end{array}$ & $\begin{array}{l}\text { Percentage of } \\
\text { species detected } \\
(\mathrm{n}=54)\end{array}$ & $\begin{array}{l}\text { Reads classified at } \\
\text { species level }(\mathrm{n}= \\
\left.7.5 \times 10^{6}\right)\end{array}$ \\
\hline taxaTarget & 99.0 & 98.9 & 97.3 & 100.0 & $3.6 \times 10^{4}$ \\
\hline EukDetect & 96.0 & 96.0 & 95.3 & 90.7 & $5.1 \times 10^{4}$ \\
\hline Metaxa2 & $66.8^{*}$ & 97.9 & 97.4 & 31.5 & $1.6 \times 10^{2}$ \\
\hline $\begin{array}{l}\text { *Metaxa2 uses a custom and older taxonomic database which often does align with the current NCBI } \\
\text { taxonomy at the family level. If the family label did not align with the current NCBI taxonomy it was } \\
\text { counted as incorrect. }\end{array}$
\end{tabular}

Table 4

Analysis of a simulated metagenome composed of 54 protist/fungal species, with reference sequences in the database of each tool. 


\begin{tabular}{|c|c|c|c|c|c|}
\hline Tool & $\begin{array}{l}\text { Precision at } \\
\text { family level } \\
(\%)\end{array}$ & $\begin{array}{l}\text { Precision at } \\
\text { genus level } \\
(\%)\end{array}$ & $\begin{array}{l}\text { Precision at } \\
\text { species level } \\
(\%)\end{array}$ & $\begin{array}{l}\text { Percent of } \\
\text { species } \\
\text { detected } \\
(n=54)\end{array}$ & $\begin{array}{l}\text { Reads classified } \\
\text { at species level } \\
\left(\mathrm{n}=7.5 \times 10^{6}\right)\end{array}$ \\
\hline taxaTarget & 99.0 & 99.0 & 97.3 & 100.0 & $3.6 \times 10^{4}$ \\
\hline EukDetect & 96.2 & 96.2 & 95.0 & 68.5 & $4.5 \times 10^{4}$ \\
\hline Metaxa2 & $76.3^{\star}$ & 99.2 & 97.0 & 31.5 & $1.3 \times 10^{2}$ \\
\hline
\end{tabular}

Table 5

Analysis of a $1 \mathrm{X}$ coverage simulated metagenome composed of 83 protist/fungal species without reference sequences in the database of the tools, but that had sequences from species related at the genus level.

\begin{tabular}{|lllllll|}
\hline Tool & $\begin{array}{l}\text { Species } \\
\text { detected at } \\
\text { family level } \\
(\mathrm{n}=83)\end{array}$ & $\begin{array}{l}\text { Species } \\
\text { detected at } \\
\text { genus level } \\
(\mathrm{n}=83)\end{array}$ & $\begin{array}{l}\text { Sensitivity } \\
\text { family }(\%)\end{array}$ & $\begin{array}{l}\text { Sensitivity } \\
\text { genus }(\%)\end{array}$ & $\begin{array}{l}\text { Precision } \\
\text { family } \\
(\%)\end{array}$ & $\begin{array}{l}\text { Precision } \\
\text { genus } \\
(\%)\end{array}$ \\
\hline taxaTarget & 81 & 79 & 97.6 & 95.2 & 94.5 & 89.1 \\
\hline EukDetect & 44 & 42 & 51.8 & 49.4 & 99.9 & 90.4 \\
\hline Metaxa2 & 23 & 14 & 27.7 & 16.9 & 90.3 & 41.6 \\
\hline
\end{tabular}

\section{Table 6}

Detection of Blastocystis in 57 Cameroonian gut samples. PCR(+) indicates that PCR detected Blastocystis in the sample. Tool(+) indicates the tool detected Blastocystis in the metagenomic sample.

\begin{tabular}{|llllll|}
\hline Tool & PCR(+) Tool(+) & PCR(-) Tool(-) & PCR(+) Tool(-) & PCR(-) Tool(+) & Accuracy (\%) \\
\hline Metaxa2 & 48 & 7 & 1 & 1 & 96.5 \\
\hline taxaTarget & 44 & 7 & 5 & 1 & 89.5 \\
\hline EukDetect & 40 & 7 & 9 & 1 & 82.5 \\
\hline
\end{tabular}


Table 7

The 20 genera detected by taxaTarget in the 57 Cameroonian gut microbiome samples 


\begin{tabular}{|c|c|c|}
\hline $\begin{array}{l}\text { Cameroon } \\
\text { (prevalence in } 57 \\
\text { samples) }\end{array}$ & $\begin{array}{l}\text { Observed in } \\
\text { human gut } \\
\text { before }\end{array}$ & Relationships to humans \\
\hline $\begin{array}{l}\text { Blastocystis } \\
(77 \%)\end{array}$ & yes & $\begin{array}{l}\text { commensal/parasite stramenopile that can consume bacteria } \\
\text { in gut [40] }\end{array}$ \\
\hline $\begin{array}{l}\text { Entamoeba } \\
(23 \%)\end{array}$ & yes & $\begin{array}{l}\text { commensal/parasitic amoeba that can consume bacteria in } \\
\text { gut [41] }\end{array}$ \\
\hline $\begin{array}{l}\text { Malassezia } \\
(19 \%)\end{array}$ & yes & broadly dispersed fungi, once thought to be restricted skin [42] \\
\hline $\begin{array}{l}\text { Saccharomyces } \\
(19 \%)\end{array}$ & yes & $\begin{array}{l}\text { broadly distributed yeast, some species are commonly found in } \\
\text { food [43] }\end{array}$ \\
\hline $\begin{array}{l}\text { Chaetoceros } \\
(12 \%)\end{array}$ & no & $\begin{array}{l}\text { none, primarily marine diatoms; might transiently occur in } \\
\text { human gut if a colonized shellfish is eaten [44] }\end{array}$ \\
\hline Pichia (7\%) & yes & yeast commonly found in food [45] \\
\hline $\begin{array}{l}\text { Strongyloides } \\
(5 \%)\end{array}$ & yes & parasitic nematode [46] \\
\hline $\begin{array}{l}\text { Trypanosoma } \\
(4 \%)\end{array}$ & yes & protist parasite [47] \\
\hline $\begin{array}{l}\text { Phytophthora } \\
(4 \%)\end{array}$ & yes & plant pathogen [48] \\
\hline Aspergillus (4\%) & yes & $\begin{array}{l}\text { diverse genus of mold consisting of several hundred } \\
\text { species [43] }\end{array}$ \\
\hline Digenea (4\%) & yes & parasitic flatworm (fluke) [49] \\
\hline $\begin{array}{l}\text { Schistosoma } \\
(4 \%)\end{array}$ & yes & parasitic flatworm (fluke) [49] \\
\hline Giardia (2\%) & yes & protist parasite [49] \\
\hline Wallemia (2\%) & no & $\begin{array}{l}\text { fungi commonly found in food; can cause skin infections; might } \\
\text { transiently occur in human gut if colonized food is } \\
\text { consumed [50] }\end{array}$ \\
\hline Perkinsus (2\%) & no & $\begin{array}{l}\text { molluscan parasite; might transiently occur in human gut if } \\
\text { colonized molluscan is consumed [51] }\end{array}$ \\
\hline Fusarium (2\%) & yes & mycotoxin-producing fungi commonly [43] found on food \\
\hline Necator (2\%) & yes & parasitic nematode [49] \\
\hline $\begin{array}{l}\text { Trepomonas } \\
(2 \%)\end{array}$ & no & $\begin{array}{l}\text { free-living, environmental protist whose ancestor might have } \\
\text { been parasitic [52] }\end{array}$ \\
\hline $\begin{array}{l}\text { Brettanomyces } \\
(2 \%)\end{array}$ & yes & yeast commonly found on fruit [45] \\
\hline Trichinella (2\%) & yes & parasitic nematode that can affect microbial composition of \\
\hline
\end{tabular}




\section{Figures}

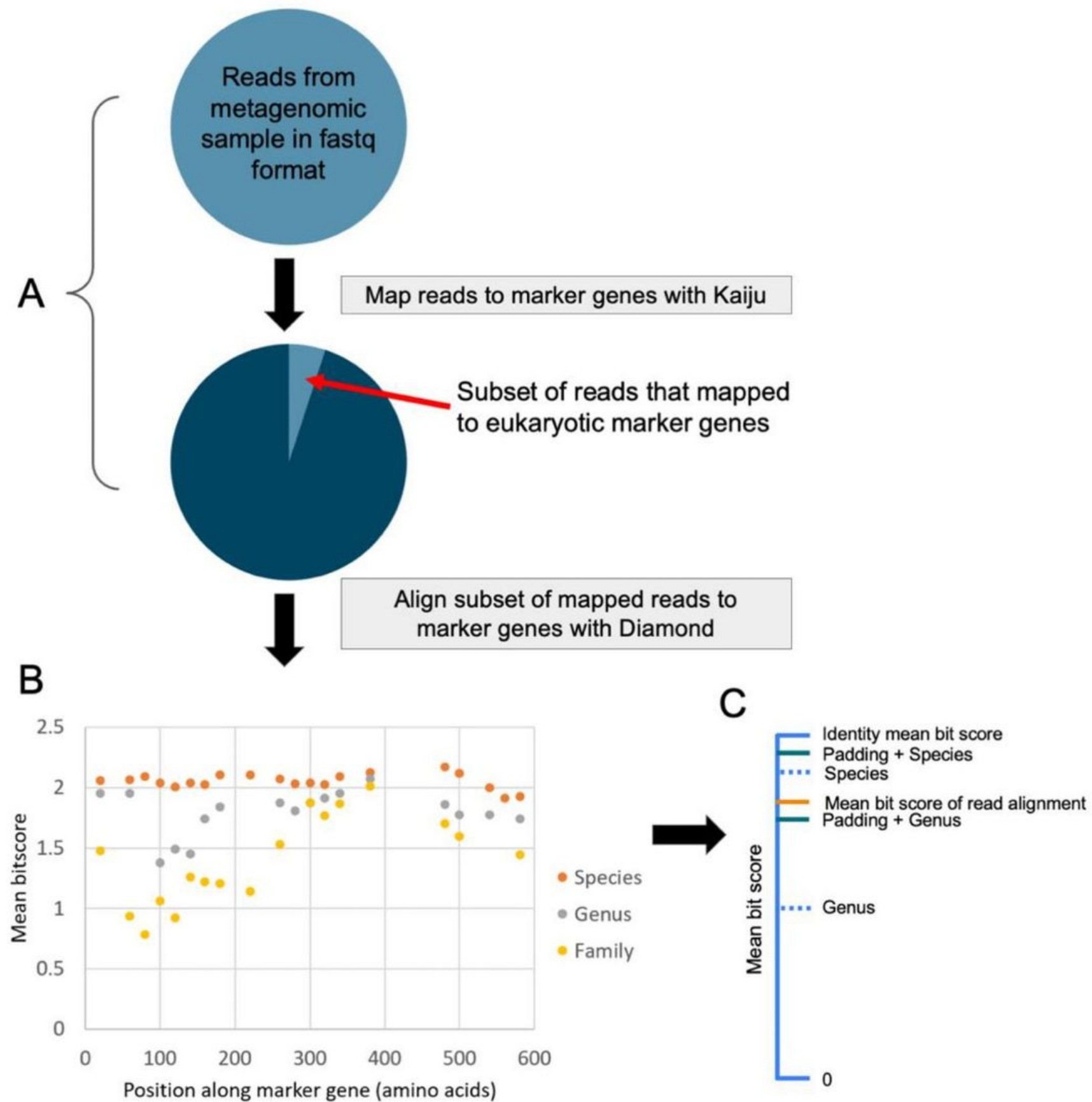

Figure 1 
Workflow diagram for taxaTarget: A) Metagenomic reads are input to taxaTarget in fastq format. The reads are first mapped to the marker genes with Kaiju-a fast, k-mer approach. The subset of reads that mapped to the marker genes with Kaiju are then more sensitively aligned to the marker genes with Diamond. The mean bit score and start position of the Diamond read alignments are used for classification. B) An example marker gene that a read mapped to. Most windows (every non-overlapping 20 amino acid region) have classification thresholds (Species, Genus, and Family) learned from training, but some regions lacked training data and thus lack thresholds. C) Classification of a read for a window where threshold padding is applied. The start position of the read alignment is used to determine the window used for classification. During training, no negative labelled reads aligned to the window at a rank broader than genus and so the thresholds for Genus and Species get padded (i.e., adjusted upwards). Here, the read gets classified as the genus of the marker gene because the mean bit score of its alignment is above Genus with padding.

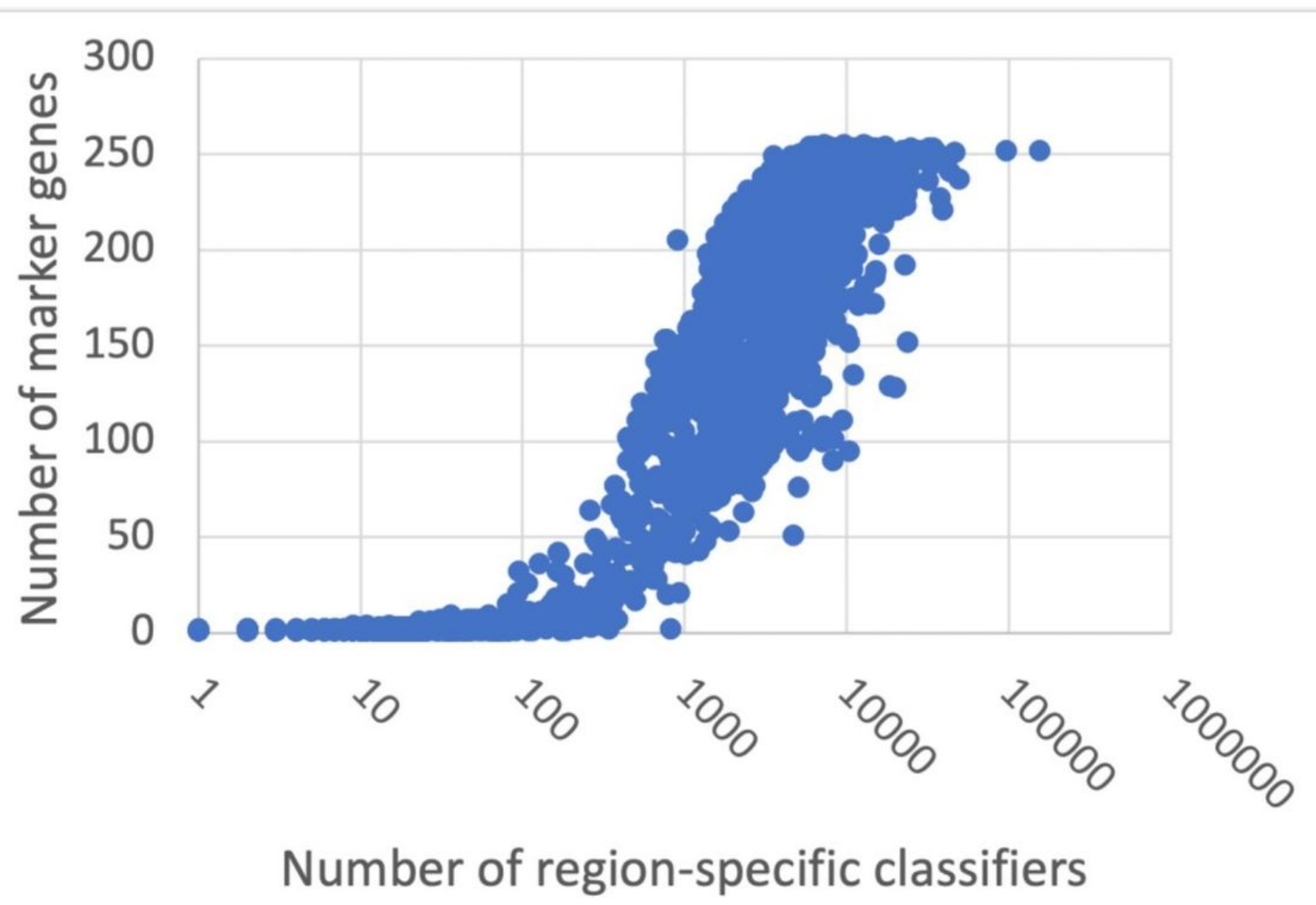

Figure 2

Scatter plot showing the number of BUSCO marker genes (255 is a complete set) versus the number of region-specific classifiers for all 4,232 species in the taxaTarget database. 


\section{Maximum taxonomic resolution of every 20 amino acid region}

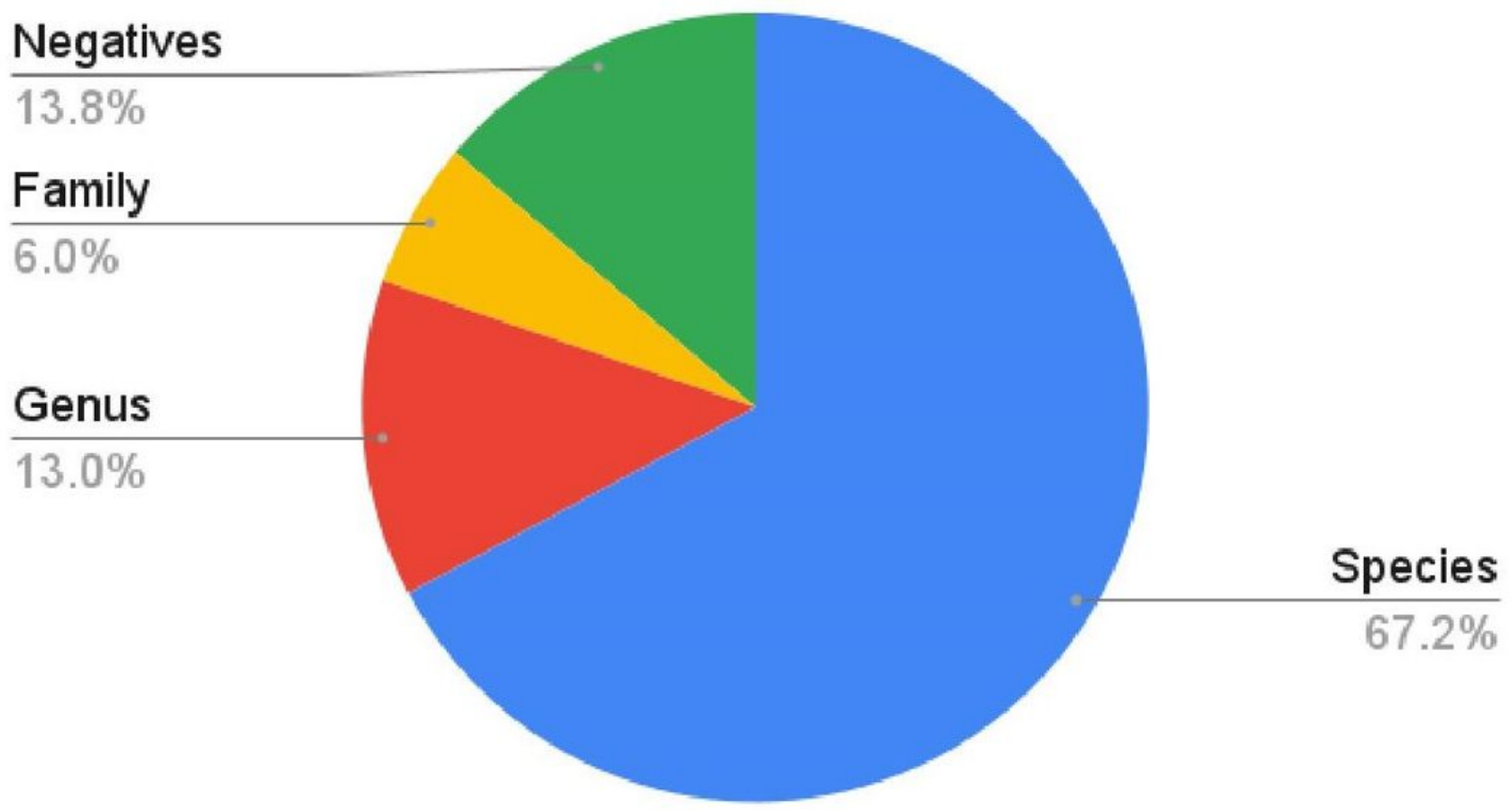

\section{Figure 3}

Potentially, there were $22.7 \times 10^{7}$ non-overlapping 20 amino acid regions (from $8.8 \times 10^{5}$ proteins) for which classifiers could be trained. The pie chart shows the maximum taxonomic resolution of the regions. Only regions where the highest bit score alignments were from species-labeled training data were used for classification in taxaTarget-about $\otimes$ of the regions.
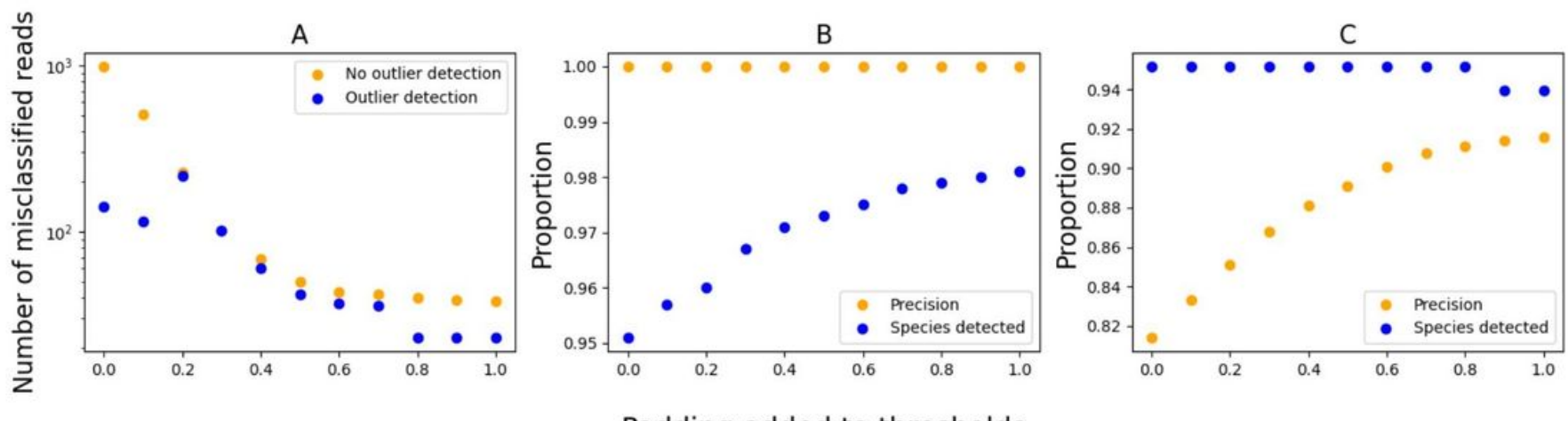

Padding added to thresholds

\section{Figure 4}

Results of parameter sweep analysis for taxaTarget. A) The non-microeukaryote metagenome was used to assess how the padding of thresholds affected the number of non-microeukaryote reads falsely 
classified as microeukaryotic (protist or fungal) species. Outlier detection refers to whether taxaTarget is set to remove marker genes with more reads mapped than expected-if sequencing randomly samples the genomes in a metagenome and that read coverage should approximate a uniform distribution. B) The microeukaryote_in_DB metagenome was used to assess the effect of threshold padding on the precision and the number of detected species $(\mathrm{n}=54)$ at the species level. $C)$ The microeukaryote_not_in_DB dataset was used to assess the effect of threshold padding on the precision and the number of detected species $(n=83)$ at the genus level.
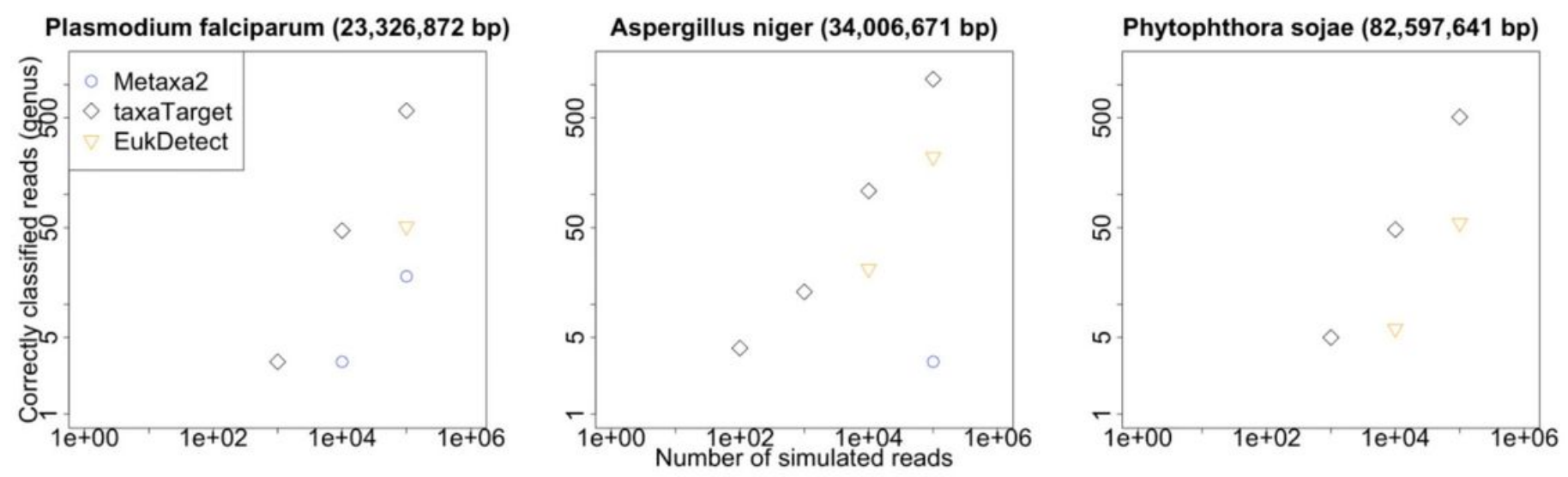

\section{Figure 5}

The sensitivity of each tool was tested with 3 simulated genomic datasets varying by 6 orders of magnitude $\left(1,10,100,1 \times 10^{3}, 1 \times 10^{4}, 1 \times 10^{5}\right)$ of reads. The datasets were simulated from the genomes of 2 protist species (Plasmodium falciparum and Phytophthora sojae) and 1 fungal species (Aspergillus niger). The genome size of each species is in parentheses besides the species name. 
Metaxa2

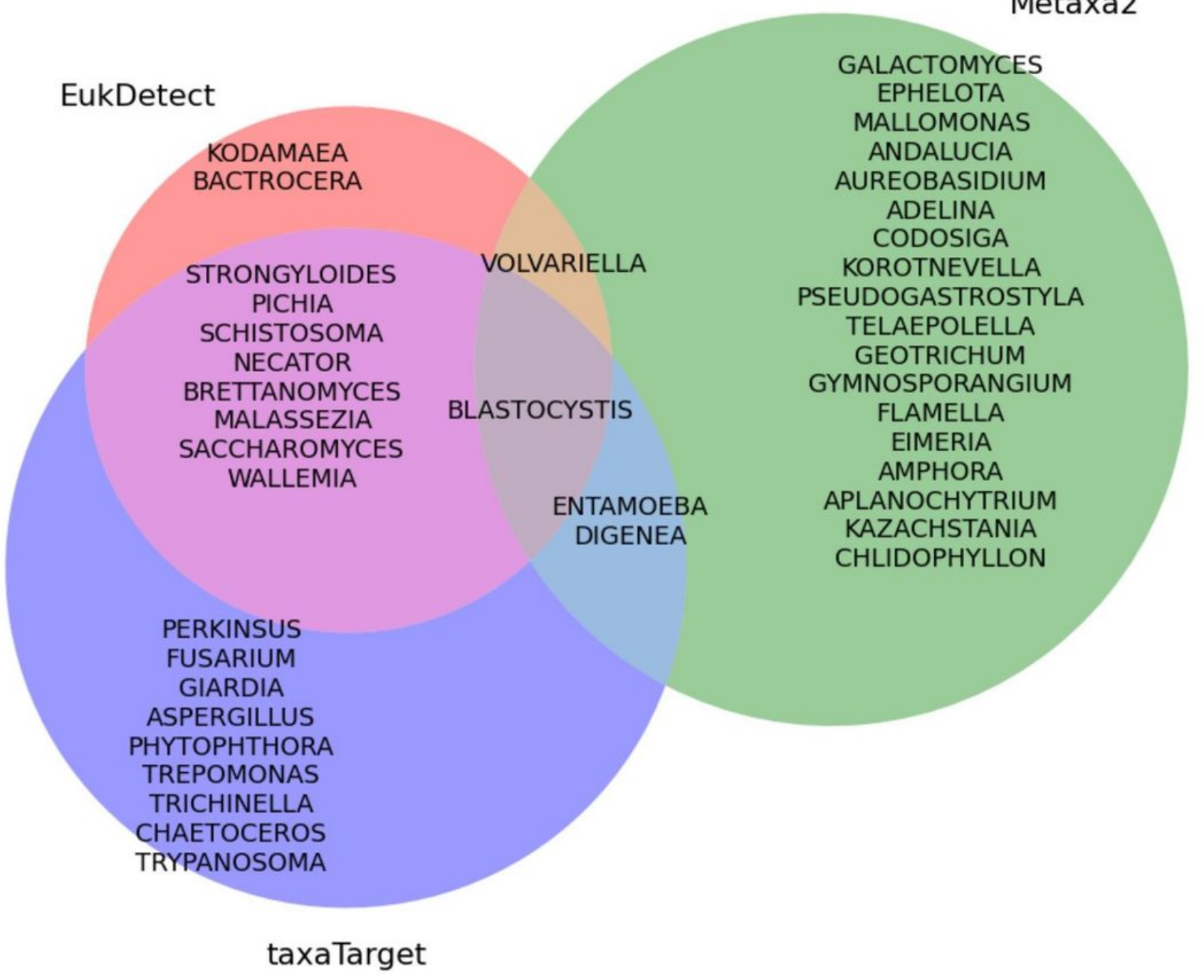

Figure 6

Genera of microeukaryotes found by the different tools in the 57 Cameroonian gut samples 
A
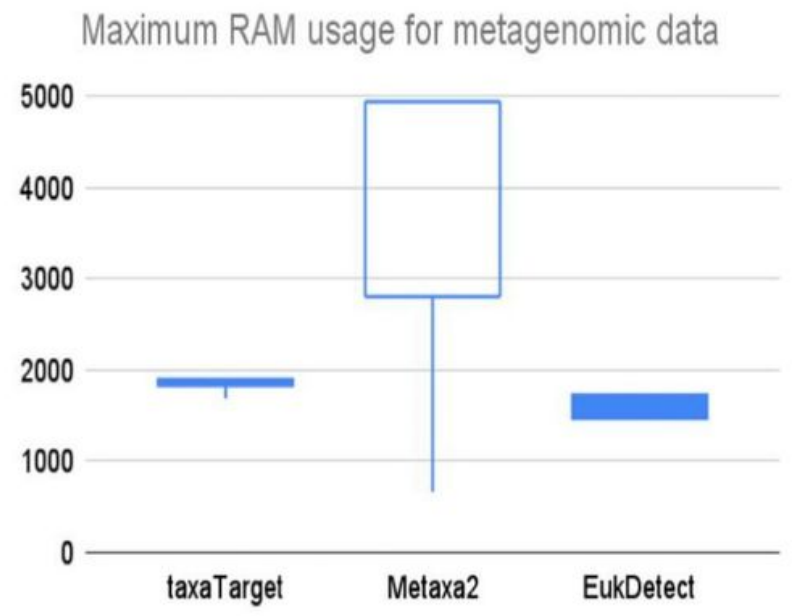

B

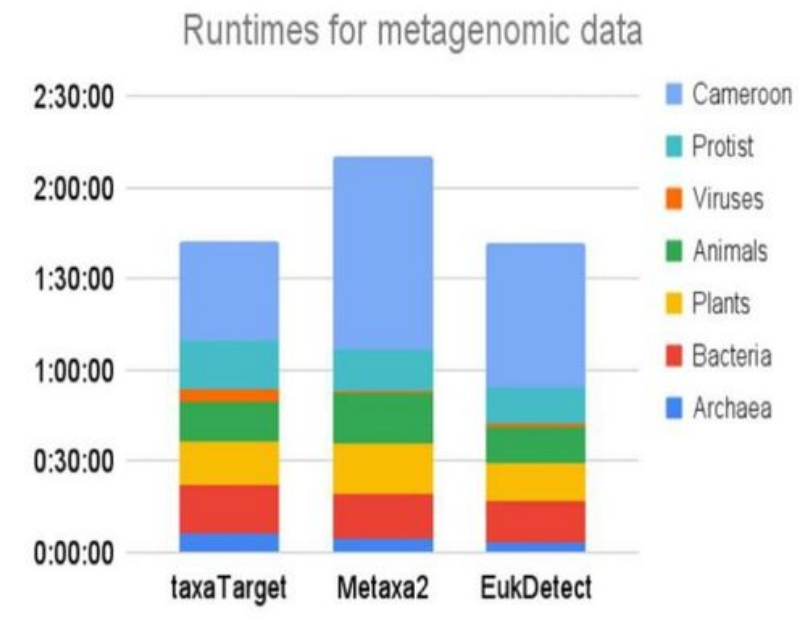

Figure 7

Runtimes and maximum RAM usage for each tool when run on several metagenomic datasets. 